\title{
A Meta-Analysis for Association of XRCC3 rs861539, MTHFR rs1801133, IL-6 rs1800795, IL-12B rs3212227, TNF- $\alpha$ rs1800629, and TLR9 rs352140 Polymorphisms with Susceptibility to Cervical Carcinoma
}

\author{
Seyedeh Fatemeh Parsaeian', Fatemeh Asadian ${ }^{2 *}$, Mojgan Karimi-Zarchi ${ }^{3,4}$, \\ Sepideh Setayesh ${ }^{5}$, Atiyeh Javaheri ${ }^{1}$, Razieh Sadat Tabatabaie ${ }^{1}$, Seyed Alireza \\ Dastgheib $^{6}$, Hossein Golestanpour ${ }^{7,8}$, Hossein Neamatzadeh ${ }^{9,10}$
}

\begin{abstract}
Background: In spite of substantial declines in both incidence and mortality rates in the past 50 years, cervical cancer remains one of the leading causes of cancer associated mortality among women globally. We performed this meta-analysis to explore the role of XRCC3 rs861539, MTHFR rs1801133, IL-6 rs1800795, IL-12B rs3212227, TNF- $\alpha$ rs1800629 and TLR9 rs352140 polymorphism with susceptibility to cervical carcinoma. Methods: The search databases include PubMed, SciELO, MedRxiv, Web of Science, Scopus, Cochrane Library, China National Knowledge Infrastructure, and China Biology Medicine disc up to 30 June 2021. The language is limited to English and Chinese. The comparison between the polymorphisms and cervical cancer was assessed using pooled odds ratio (OR) and $95 \%$ confidence interval (CI). The data are statistically analyzed by Comprehensive Meta-Analysis (CMA) 2.0 software. Results: A total of 59 studies including seven studies with 1,112 cases and 1,233 controls on XRCC 3 rs861539, 14 studies with 2,694 cases and 3349 controls MTHFR rs1801133, four studies with 1,121 cases and 1,109 controls on IL-12B rs3212227, seven studies with 1,452 cases and 2,186 controls on IL-6 rs1800795, 20 studies with 4,781 cases and 4909 controls on TNF- $\alpha$ rs 1800629 , and seven studies with 1743 cases and 2292 controls on TLR9 rs 352140 were included. There was a significant association between XRCC3 RS861539, TNF- $\alpha$ rs 1800629, and IL-6 rs1800795 polymorphisms and an increased risk of cervical carcinoma in overall population. However, the MTHFR rs1801133, IL-12B rs3212227 and TLR9 rs352140 polymorphisms were not associated. Conclusion: The pooled analysis showed that XRCC3 RS861539, TNF- $\alpha$ rs1800629, and IL-6 rs1800795 were associated with cervical carcinoma susceptibility, but not MTHFR rs1801133, IL-12B rs3212227 and TLR9 rs352140 polymorphisms.
\end{abstract}

Keywords: Cervical carcinoma- cervical cancer- meta-analysis- gene- polymorphism

Asian Pac J Cancer Prev, 22 (11), 3419-3431

\section{Introduction}

Cancer is one of the main public health problems globally with about 18.1 million new cancer cases and 9.6 million cancer deaths in 2018 (Ghelmani et al., 2021; Jarahzadeh et al., 2021; Antikchi et al., 2021). The World Health Organization (WHO) states cervical cancer is the second most frequent cancer among women worldwide
(Hamadani et al., 2017; Da Silva et al., 2021) with an estimated 570,000 new cases and 311,000 deaths in 2018 worldwide (Yi et al., 2020). It is reported that $85 \%$ of cervical cancer cases and $87 \%$ of the cervical cancer deaths occur in less developed countries (WHO/ICO, 2010). With over 500,000 cases of cervical cancer reported each year, nearly 80 percent of those are in developing countries, including Africa with 68,000, an estimated 77,000 in Latin

${ }^{1}$ Department of Obstetrics and Gynecology, Shahid Sadoughi University of Medical Sciences, Yazd, Iran. ${ }^{2}$ Department of Medical Laboratory Sciences, School of Paramedical Science, Shiraz University of Medical Sciences, Shiraz, Iran. ${ }^{3}$ Endometriosis Research Center, Iran University of Medical Sciences, Tehran, Iran. ${ }^{4}$ Department of Obstetrics and Gynecology, Firoozgar Hospital, Iran University of Medical Sciences, Tehran, Iran. ${ }^{5}$ School of Medicine, Shiraz University of Medical Sciences, Shiraz, Iran. ${ }^{6}$ Department of Medical Genetics, School of Medicine, Shiraz University of Medical Sciences, Shiraz, Iran. ${ }^{7}$ Department of Genetics, Marvdasht Branch, Azad University, Marvdasht, Iran. ${ }^{8}$ Biotechnology Research Center, International Campus, Shahid Sadoughi University of Medical Sciences, Yazd, Iran. ${ }^{9}$ Mother and Newborn Health Research Center, Shahid Sadoughi University of Medical Sciences, Yazd, Iran. ${ }^{10}$ Department of Medical Genetics, Shahid Sadoughi University of Medical Sciences, Yazd, Iran. *For Correspondence: fasadian.pat@gmail.com 
America and the Caribbean, and 245,000 in Asia (Arbyn et al., 2020). Cervical cancer is driven by persistent infection with one of 15 carcinogenic human papillomavirus (HPV) types (Ghaemmaghamiet al., 2008; Chen et al., 2011). However, the impact of HPV type and intratypic variants on patient outcomes is far less understood (Karimi-Zarchi et al., 2013; Baghestani et al., 2018; Rader et al., 2019).

To date, the occurrence and development of cervical cancer is suggested to be associated with persistent HPV infection (Ghaemmaghami et al., 2008; Zarchi et al., 2010; Pal and Kundu, 2020). The DNA of HPV integrates into the host cell genome and disrupts the open reading frame and causes overexpression of E6 and E7 genes (Binesh et al., 2012; Soheili et al., 2016). However, the specific molecular mechanisms and potential single gene require further studies. Familial based studies and evaluation of inherited genetic variations revealed that host genetic factors have a role in cervical cancer pathogenesis (Yang et al., 2020; Ahmadi et al., 2021). Recently, a comprehensive review study showed that CDK1, CCNB1, ITGB1, FN1, MMP9 and STAT1 played different roles in the progression of cervical cancer through different signaling pathways (Sayad, Ahmadi, Nekouian, et al., 2020; Yi et al., 2020). Moreover, studies have shown that cervical cancer has a heritable genetic component. However, as other complex disease, the identified loci only explain a minority of the risk of cervical cancer. This gap of the heritability explained by genetic markers genome-wide association studies and the heritability identified in familial studies has been termed as 'missing heritability' (Chen et al., 2016; Leo et al., 2017; Sayad, Ahmadi, Moradi, et al., 2020). Thus, our understanding of the genetic basis of cervical cancer is still limited. In this meta-analysis, we explore the role of XRCC3 rs861539, MTHFR rs1801133, IL-6 rs1800795, IL-12B rs3212227, TNF- $\alpha$ rs1800629, and TLR9 rs352140 polymorphisms in susceptibility to cervical cancer.

\section{Materials and Methods}

\section{Literate Search Strategy}

We performed a comprehensive literature search on PubMed, Scopus, China National Knowledge Infrastructure, Wanfang, VIP Information Chinese Journal Service Platform, and China Biology Medicine disc, PubMed, EMBASE, Web of Science and the Cochrane Library, Google Scholar, Cochrane Library, EMBASE, Scientific Information Database (SID), WanFang, VIP, Chinese Biomedical Database (CBD), Scientific Electronic Library Online (SciELO), China National Knowledge Infrastructure (CNKI), IranDoc and Egyptian Knowledge Bank (EKB) Journals database to identify all relevant studies on the association of XRCC3 rs861539, MTHFR rs1801133, IL-6 rs1800795, IL-12B rs3212227, TNF- $\alpha$ rs1800629 and TLR9 rs352140 with cervical cancer up to 30th June 2021. The following terms were used in various combinations in this search: " Uterine Cervical Neoplasm " OR 'Cervix Cancer" OR 'Cervical Cancer" OR “'Cervical Neoplasm” OR 'Cervical Carcinoma” OR 'Squamous Cell Carcinoma" OR “'Adenocarcinoma") AND ("X-Ray Repair Cross Complementing 3" OR

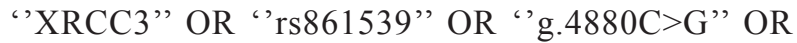
'c.-237C >G" OR 'n.54-321G>C") AND ("Methylene tetrahydrofolate reductase", OR ' MTHFR" OR “'677C>T" OR “'rs1801133” OR “'g.14783C>T" OR “c.788C>T" OR “"p.Ala263Val" OR "p.Ala222Val") AND ("Interleukin 12" OR "IL-12" OR "'1188A>C" OR "'rs3212227" OR " g.158742950T $>\mathrm{G}$ " OR “" g.19532A>C" OR " c.*159A>C") AND ('Interleukin 6" OR "'IL-6" OR "'-174G>C" OR "'rs1800795" OR " g.4880C $>$ G" OR 'c.-274C $>$ G" OR " n.54-321G $>C$ ") AND ("Toll like receptors" OR " TLR" "'OR "rs352140" OR "'2848G/A") AND ("Gene" OR "'Single-Nucleotide Polymorphism" or "SNP" OR "Polymorphism" OR "'Genotype' OR "Allele" OR " Variant" OR "Variation" OR "Mutation" OR 'Mutant"). The search was carried out in English, Chinese and Persian. When overlapping data on the same cases were included in more than one publication, only the one with the larger sample size was selected. Moreover, the reference list of the retrieved studies and reviews manually checked to identify more potential eligible studies.

\section{Eligibility criteria}

To obtain the papers, the following criteria should be met to include the papers in our study: 1) studies with case-control or cohort design; 2) studies reported original data; 3) studies appraised association of XRCC3 rs861539, MTHFR rs1801133, IL-6 rs1800795, IL-12B rs3212227, TNF- $\alpha$ rs1800629 and TLR9 rs352140 polymorphisms with cervical cancer; and 4) studies with available and sufficient data for calculating an odds ratio (OR) with 95\% confidence interval (CI). The following exclusion criteria were also used: 1) studies on other polymorphism at XRCC3, MTHFR, IL-12B, IL-6, and TNF- $\alpha$ genes; 2) animal studies or in vitro studies; 3 ) studies with in sufficient data on genotype frequencies or which the number of genotypes and alleles could not be ascertained; 4) linkage studies; 5) family based studies including sibling, twins and trios-parents studies; 6) abstracts, case reports, commentaries, editorials, conference articles, reviews, proceedings and meta-analyses; and 7) duplicates studies or overlapping data.

\section{Data Extraction}

Two authors reviewed and extracted necessary information independently in accordance with our inclusion criteria. For conflicting data, the authors carried out discussions until a consensus was reached. If they could not reach a consensus, disagreement was resolved by the third author who participated in the discussion. For each eligible study the following data was collected: first author name, year of publication, country of origin, ethnicity (Caucasian, Asian, African, Mixed populations), genotyping methods, sample size, allele and genotype frequency of XRCC3 rs861539, MTHFR rs1801133, IL-12B rs3212227, IL-6 rs1800795, TNF- $\alpha$ rs1800629 and TLR9 rs352140 polymorphisms in cervical cancer cases and controls, Minor Allele Frequency (MAFs) and Hardy-Weinberg equilibrium (HWE) in healthy controls. In this meta-analysis different case-control groups or cohorts in one publication were considered as independent 
studies. We did not define any minimum sample size to include in this meta-analysis. The "mixed" group means mixed or unknown populations. If more than one study was published by the same author(s) using repeated or overlapped data, the studies with the largest sample size or the most recently published study was included to the meta-analysis. If selected articles did not reported necessary data the corresponding authors was contacted by email to request the missing data.

\section{Statistical Analysis}

The comparison between the XRCC3 rs861539, MTHFR rs1801133, IL-6 rs1800795, IL-12B rs3212227, TNF- $\alpha$ rs1800629 and TLR9 rs352140 polymorphism and cervical cancer was assessed using pooled odds ratio (OR) and 95\% confidence interval (CI). The pooled data were calculated under five genetic models, i.e., allele (B vs. A), homozygote (BB vs. AA), heterozygote (BA vs. $\mathrm{BB})$, dominant $(\mathrm{BB}+\mathrm{BA}$ vs. $\mathrm{AA})$ and recessive $(\mathrm{BB}$ vs. $\mathrm{BA}+\mathrm{AA}$ ), in which a " $\mathrm{A}$ " denotes a major allele; "B" denotes a minor allele. The $\chi^{2}$ test and $\mathrm{I}^{2}$ statistics were used to assess whether there was between-study heterogeneity, in which $\mathrm{P}>.10$ and $\mathrm{I}^{2}<50 \%$ could be considered that there was no statistical heterogeneity between the research results. The fixed effect model was selected for data consolidation; $\mathrm{P} \leq .10$ and $\mathrm{I}^{2} \geq 50 \%$ could be considered that there was statistical heterogeneity between the research results, and a random effect model was used for data consolidation. If there was significant heterogeneity among studies, the random effects model (DerSimonian and Laird) was used; otherwise, the fixedeffects model (Mantel and Haenszel) was acceptable. The Hardy-Weinberg equilibrium of the control group was evaluated using the $\chi^{2}$ test, and the expected and actual genotype frequencies of the control group were compared. In this meta-analysis, P-values of $\leq .05$ were considered statistically significant (Azadi-Yazdi et al., 2017; Mirjalili et al., 2019; Dastgheib et al., 2020). In addition, the sensitivity analysis was performed by excluding HWE-violating studies. Potential publication bias was evaluated using the Egger's linear regression test and Begg's quantitative test. The asymmetric plot of Egger's test and the P-value of Begg's test less than 0.05 were considered a significant publication bias. If there was evidence of publication bias $(\mathrm{P}<0.05)$, trim and fill method was applied to adjust for the effect of publication bias (Jafari et al., 2020). All statistical analyses were performed using Comprehensive Meta-Analysis (CMA) Software version 2.0 (Biostat, Englewood, USA). All tests were two-sided, and the $\mathrm{P}<0.05$ was considered statistically significant.

\section{Results}

\section{Characteristics of the Enrolled Studies}

The flowchart of selection of studies and reasons for exclusion is presented in Figure 1. There were 1,013 articles relevant to our search words and 395 duplicated studies were excluded. Then, 207 studies removed after reading titles and abstracts. Following the inclusion exclusion criteria 352 studies were excluded. Finally, a total of 59 studies were included in this meta-analysis. There are seven studies with 1112 cases and 1233 controls on XRCC3 rs861539 (He et al., 2008; Xiao, 2009; Settheetham-Ishida et al., 2011; Djansugurova et al., 2013; Pérez et al., 2013; Al-Harbi et al., 2017), 14 studies with 2694 cases and 3349 controls MTHFR rs1801133 (Lambropoulos et al., 2003; Sull et al., 2004; Kang et al., 2005; Zoodsma et al., 2005; Delgado-Enciso et al., 2006; Ma et al., 2006; Nandan et al., 2008; Shekari et al., 2008; Kohaar et al., 2010; Yang et al., 2011; Prasad and Wilkhoo, 2011; Tong et al., 2011; Mostowska et al., 2011; von Keyserling et al., 2011), four studies with 1121 cases and 1109 controls on IL-12B rs3212227 (Chen et al., 2009; Do Carmo Vasconcelos De Carvalho et al., 2012; Roszak, Mostowska, et al., 2012), seven studies with 1452 cases and 2186 controls on IL-6 rs1800795 (Nogueira De Souza et al., 2006; Gangwar et al., 2009; Grimm et al., 2011; Shi et al., 2013; de Lima Júnior et al., 2016; Pu et al., 2016; Zidi et al., 2017), 20 studies with 4,781 cases and 4909 controls on TNF- $\alpha$ rs 1800629 (Stanczuk et al., 2019; Jang et al., 2001; Calhoun et al., 2002; Gostout et al., 2003; Deshpande et al., 2005; Duarte et al., 2005; Govan et al., 2006; Kohaar et al., 2007; Singh et al., 2009; Zu et al., 2010; Ivansson et al., 2010; Zuo et al., 2011; Wang et al., 2011, 2012; Badano et al., 2012; Barbisan et al., 2012; Sousa et al., 2014; Roszak et al., 2015; Zidi et al., 2015) and seven studies with 1743 cases and 2292 controls on TLR9 rs352140 (Pandey et al., 2011; Roszak, Lianeri, et al., 2012; Lai et al., 2013; Bi, 2014; Zidi et al., 2016; Jin et al., 2017). The details of included studies were shown in Tables 1 and 2. All those 22 studies were reported in English and Chinese. Among those 59 studies, 31 studies were from Asian populations, 18 studies from Caucasian populations, five studies from African populations, and five studies were from mixed populations. The studies have been carried out in China, Thailand, Argentina, Kazakhstan, Brazil, Saudi Arabia, Greece, Korea, Netherlands, Mexico, India, Poland, Germany, Austria, Tunisia, Zimbabwe, USA, South Africa, Sweden, and Portugal. The sample size of cases ranged from 21 to 636, while the sample size of controls ranged from 73 to 800 in the controls. Eight different methods including AS-PCR, PCR-RFLP, Direct Sequencing, SnapShot, TaqMan, LDR-PCR, ARMS-PCR, qRT-PCR and HMR were used to genotyping those polymorphisms. HardyWeinberg equilibrium (HWE) test was calculated for all publications and $\mathrm{P}<0.05$ was considered as a departure from HWE (Table 1).

\section{Quantitative Data Synthesis XRCC3 RS861539}

The summary for the association of XRCC3 RS861539 polymorphism with cervical cancer risk are shown in Table 3. Pooled data revealed that XRCC3 RS861539 polymorphism was significantly associated with susceptibility to cervical cancer under the heterozygote genetic model (TC vs. CC: OR $=1.00,95 \%$ CI 1.066-1.585, $\mathrm{p}=0.009$ ). Moreover, stratified analysis by ethnicity revealed that the polymorphisms was significantly associated with cervical cancer in Asian women under three genetic models, i.e., allele (T vs. C: $\mathrm{OR}=1.302$, 


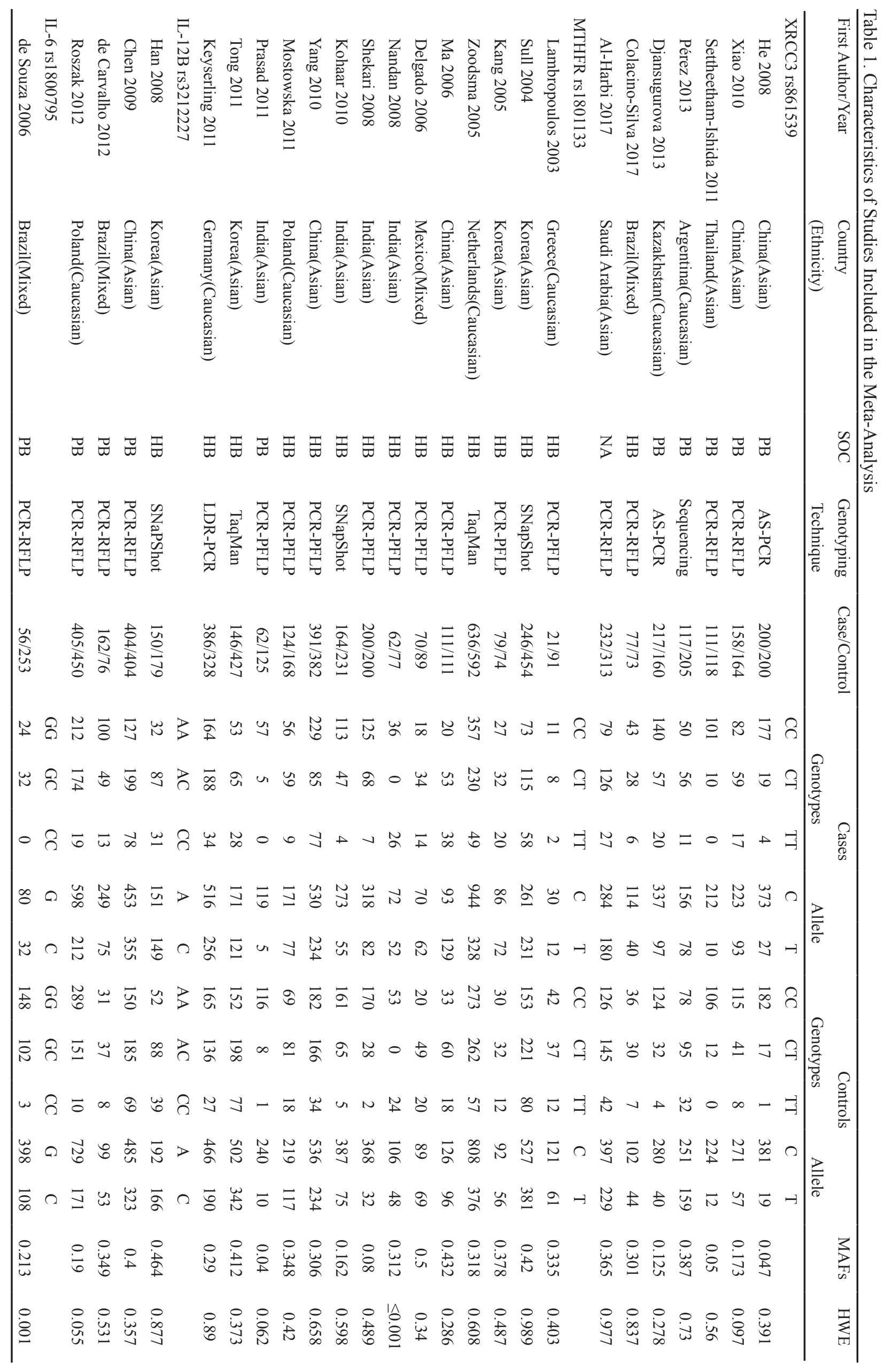

3422 Asian Pacific Journal of Cancer Prevention, Vol 22 
95\% CI 1.076-1.576, $\mathrm{P}=0.007$ ), heterozygote (TC vs. $\mathrm{CC}: \mathrm{OR}=1.441,95 \% \mathrm{CI} 1.113-1.867, \mathrm{p}=0.006)$ and dominant (TT+TC vs. CC: OR $=1.469,95 \%$ CI 1.148 $1.880, \mathrm{p}=0.002)$, but not in Caucasian women.

\section{$T N F-\alpha$ rs 1800629}

The summary for the association of TNF- $\alpha$ rs 1800629 polymorphism with cervical cancer risk are shown in Table 3. Pooled data from all eligible studies indicated that the TNF- $\alpha$ rs1800629 polymorphism was associated with cervical cancer risk in overall population under four genetic models i.e., allele (A vs. $\mathrm{G}$ : $\mathrm{OR}=1.277$, 95\% CI $=1.104-1.477, \mathrm{P}=0.001$ ), homozygote (AA vs. $\mathrm{GG}: \mathrm{OR}=1.333,95 \% \mathrm{CI}=1.062-1.674, \mathrm{P}=0.013)$, heterozygote (AG vs. GG: $\mathrm{OR}=1.307,95 \% \mathrm{CI}=1.064$ $1.605, \mathrm{P}=0.011$ ), and dominant (AA+AG vs. $\mathrm{GG}: \mathrm{OR}=$ $1.324,95 \% \mathrm{CI}=1.104-1.587, \mathrm{P}=0.002$ ). The subgroup analysis by ethnicity also showed that this polymorphism was associated with cervical cancer in Caucasian women under three genetic models i.e., allele (A vs. G, OR = $1.242,95 \% \mathrm{CI}=1.043-1.478, \mathrm{P}=0.015)$; homozygote (AA vs. $\mathrm{GG}, \mathrm{OR}=1.586,95 \% \mathrm{CI}=1.147-2.193, \mathrm{P}=$ 0.005 ), and recessive (AA vs. $\mathrm{AG}+\mathrm{GG}, \mathrm{OR}=1.569,95 \%$ $\mathrm{CI}=1.137-2.165, \mathrm{P}=0.006)$ and in African women under two genetic models i.e., heterozygote (AG vs. GG, OR = $1.670,95 \% \mathrm{CI}=1.228-2.270, \mathrm{P}=0.001)$ and dominant (AA+AG vs. GG, OR =1.453, 95\% CI = 1.111-1.902, $\mathrm{P}$ $=0.006)$, but not in Asian women.

\section{MTHFR rs 1801133}

The summary for the association of MTHFR rs1801133 polymorphism with cervical cancer risk are shown in Table 3. Pooled results showed that the MTHFR rs1801133 polymorphism was not associated with cervical cancer in overall population and by ethnicity.

\section{IL-12B rs3212227}

The summary for the association of IL-12B rs3212227 polymorphism with cervical cancer risk are shown in Table 4. Pooled data showed that the IL-12B rs3212227 polymorphism was not associated with cervical cancer globally. Stratified analysis by ethnicity revealed that this polymorphism was associated with cervical cancer in Asian women under two genetic models i.e., heterozygote (CA vs. AA: $\mathrm{OR}=1.349,95 \%$ CI 1.032-1.762, $\mathrm{p}=0.028$ ) and dominant $(\mathrm{CC}+\mathrm{CA}$ vs. $\mathrm{AA}: \mathrm{OR}=1.340,95 \% \mathrm{CI}$ $1.041-1.725, \mathrm{p}=0.023)$.

\section{IL-6 rs 1800795}

The summary for the association of IL-6 rs1800795 polymorphism with cervical cancer risk are shown in Table 4. Pooled data from all eligible studies showed that the IL-6 rs1800795 polymorphism was significantly associated with cervical cancer risk under four genetic models, i.e., allele (C vs. G: OR $=1.294,95 \%$ CI 1.071$1.564, \mathrm{p}=0.007)$, homozygote (CC vs. GG: OR $=1.633$, 95\% CI 1.059-2.520, $\mathrm{p}=0.027)$, dominant (CC+CG vs. $\mathrm{GG}: \mathrm{OR}=1.312,95 \%$ CI $1.048-1.643, \mathrm{p}=0.018)$ and recessive ( $\mathrm{CC}$ vs. $\mathrm{CG}+\mathrm{GG}: \mathrm{OR}=1.592,95 \% \mathrm{CI}$ $1.268-1.999, \mathrm{p} \leq 0.001)$. Moreover, subgroup analysis by 


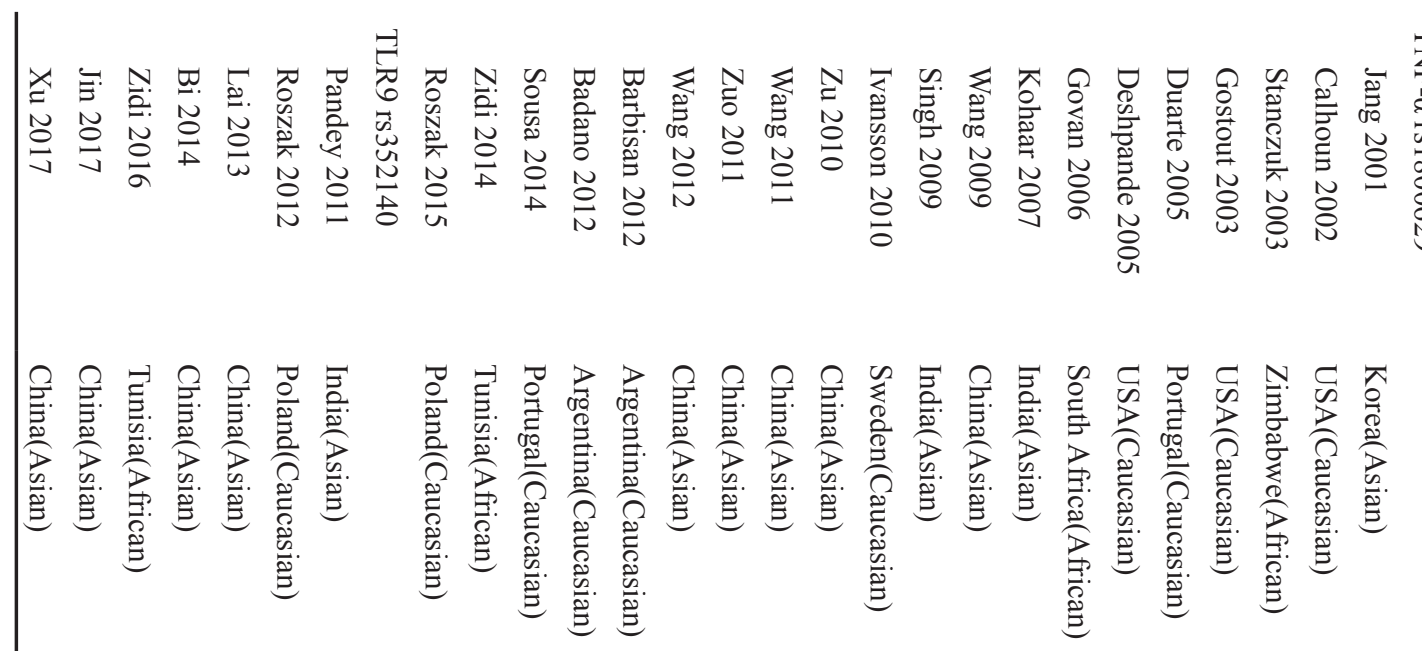

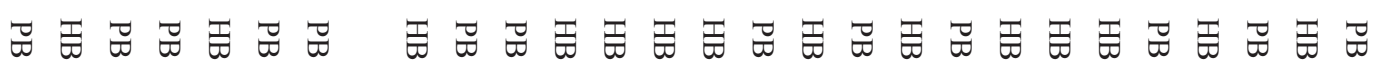

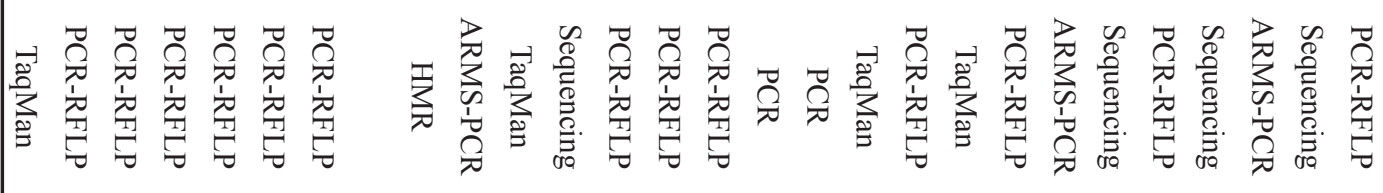

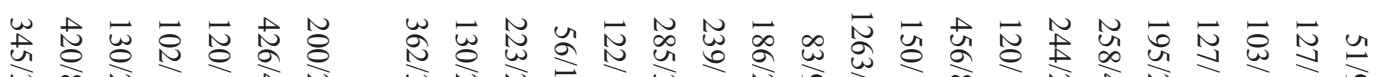

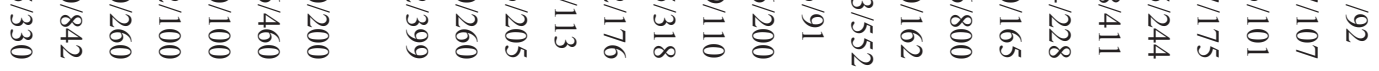

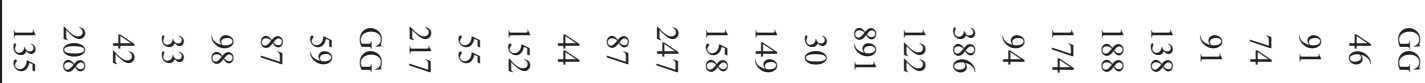

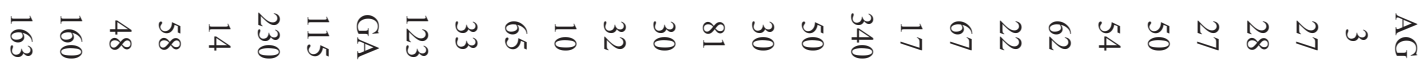

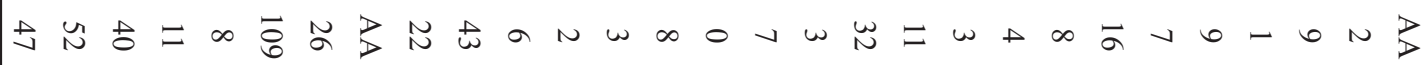

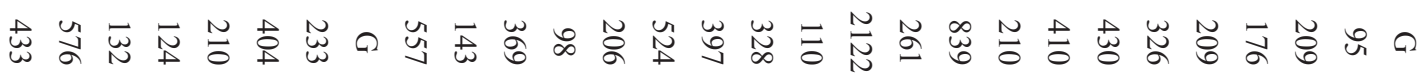

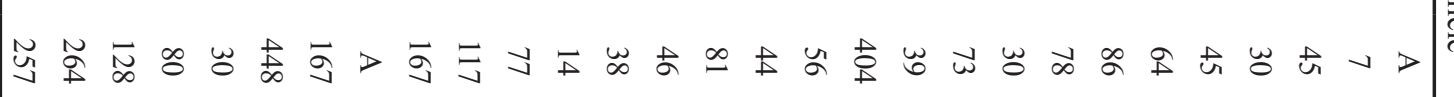

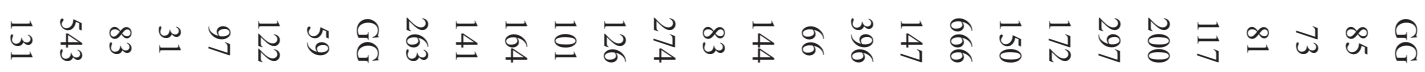

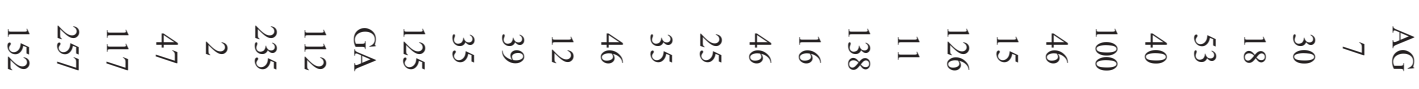

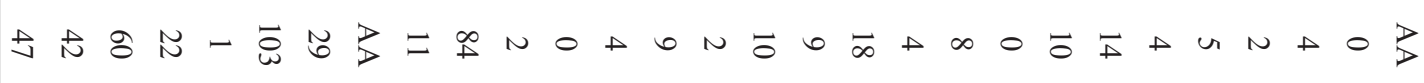

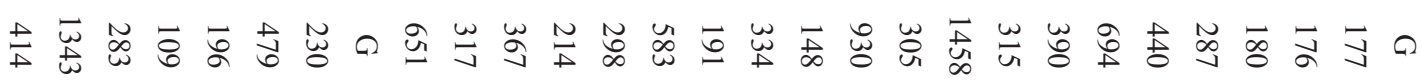

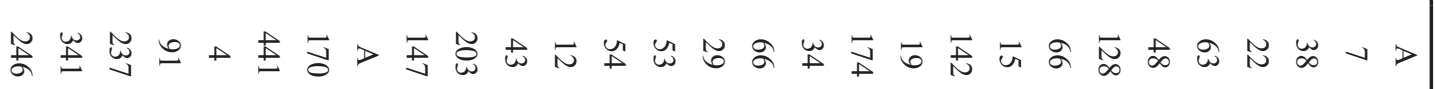

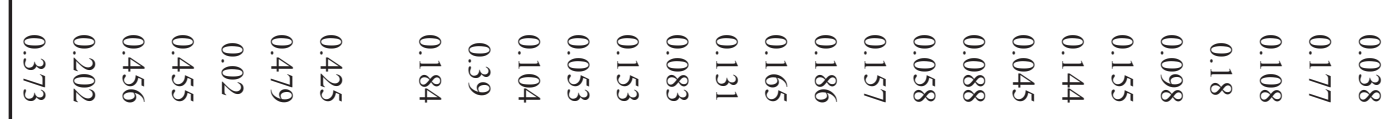
岀 
DOI:10.31557/APJCP.2021.22.11.3419

Genetic Variants and Cervical Carcinoma

Table 3. Results of the Association of XRCC3 RS861539, MTHFR rs1801133 and IL-12B rs3212227 Polymorphisms with Cervical Cancer Risk

\begin{tabular}{|c|c|c|c|c|c|c|c|c|c|c|}
\hline \multirow[t]{2}{*}{ Subgroup } & \multirow[t]{2}{*}{ Genetic Model } & \multirow{2}{*}{$\begin{array}{l}\text { Type of } \\
\text { Model }\end{array}$} & \multicolumn{2}{|c|}{ Heterogeneity } & \multicolumn{2}{|c|}{ Odds Ratio } & \multirow[b]{2}{*}{$Z_{\text {test }}$} & \multirow[b]{2}{*}{$\mathrm{P}_{\mathrm{OR}}$} & \multicolumn{2}{|c|}{ Publication Bias } \\
\hline & & & $I^{2}(\%)$ & $\mathrm{P}_{\mathrm{H}}$ & OR & $95 \% \mathrm{CI}$ & & & $\mathrm{P}_{\mathrm{Beggs}}$ & $\mathrm{P}_{\text {Eggers }}$ \\
\hline \multicolumn{11}{|c|}{ XRCC3 rs861539 } \\
\hline \multirow[t]{5}{*}{ Overall } & T vs. C & Random & 73.82 & 0.001 & 1.223 & $0.897-1.669$ & 1.272 & 0.203 & 1 & 0.901 \\
\hline & TT vs. CC & Random & 68.44 & 0.007 & 1.456 & $0.723-2.932$ & 1.053 & 0.292 & 0.707 & 0.376 \\
\hline & TC vs. CC & Fixed & 26.84 & 0.224 & 1.3 & $1.066-1.585$ & 2.596 & 0.009 & 0.548 & 0.242 \\
\hline & $\mathrm{TT}+\mathrm{TC}$ vs. CC & Random & 58.55 & 0.025 & 1.27 & $0.935-1.726$ & 1.53 & 0.126 & 0.763 & 0.452 \\
\hline & TT vs. TC+CC & Random & 64.54 & 0.015 & 1.309 & $0.693-2.470$ & 0.829 & 0.407 & 0.452 & 0.225 \\
\hline \multirow[t]{5}{*}{ Asians } & T vs. C & Fixed & 60.39 & 0.056 & 1.302 & $1.076-1.576$ & 2.716 & 0.007 & 1 & 0.862 \\
\hline & TT vs. CC & Fixed & 58.94 & 0.088 & 1.457 & $0.918-2.314$ & 1.595 & 0.111 & 1 & 0.446 \\
\hline & TC vs. CC & Fixed & 14.62 & 0.319 & 1.441 & $1.113-1.867$ & 2.768 & 0.006 & 0.308 & 0.474 \\
\hline & $\mathrm{TT}+\mathrm{TC}$ vs. CC & Fixed & 36.18 & 0.195 & 1.469 & $1.148-1.880$ & 3.055 & 0.002 & 0.734 & 0.666 \\
\hline & TT vs. TC+CC & Fixed & 61.31 & 0.075 & 1.165 & $0.754-1.801$ & 0.689 & 0.491 & 1 & 0.375 \\
\hline \multirow[t]{5}{*}{ Caucasians } & T vs. $\mathrm{C}$ & Random & 91.87 & 0 & 1.253 & $0.500-3.138$ & 0.481 & 0.63 & NA & NA \\
\hline & TT vs. CC & Random & 89.45 & 0.002 & 1.484 & $0.188-11.730$ & 0.374 & 0.708 & NA & NA \\
\hline & TC vs. CC & Fixed & 45.46 & 0.176 & 1.234 & $0.873-1.743$ & 1.193 & 0.233 & NA & NA \\
\hline & $\mathrm{TT}+\mathrm{TC}$ vs. CC & Random & 83.94 & 0.013 & 1.248 & $0.551-2.826$ & 0.532 & 0.595 & NA & NA \\
\hline & TT vs. TC+CC & Random & 88.24 & 0.004 & 1.425 & $0.210-9.655$ & 0.363 & 0.716 & NA & NA \\
\hline \multicolumn{11}{|c|}{ MTHFR rs1801133 } \\
\hline \multirow[t]{5}{*}{ Overall } & T vs. C & Random & 73.78 & $\leq 0.001$ & 1.132 & $0.956-1.341$ & 1.434 & 0.151 & 0.62 & 0.232 \\
\hline & TT vs. CC & Random & 49.57 & 0.013 & 1.212 & $0.924-1.590$ & 1.388 & 0.165 & 0.964 & 0.802 \\
\hline & TC vs. CC & Random & 77.11 & $\leq 0.001$ & 0.985 & $0.755-1.284$ & -0.113 & 0.91 & 0.843 & 0.438 \\
\hline & $\mathrm{TT}+\mathrm{TC}$ vs. $\mathrm{CC}$ & Random & 79.6 & $\leq 0.001$ & 1.095 & $0.842-1.423$ & 0.677 & 0.498 & 0.752 & 0.215 \\
\hline & TT vs. TC+CC & Random & 83.79 & $\leq 0.001$ & 1.41 & $0.913-2.176$ & 1.551 & 0.121 & 0.62 & 0.867 \\
\hline \multicolumn{11}{|l|}{ Ethnicity } \\
\hline \multirow[t]{5}{*}{ Caucasians } & T vs. $\mathrm{C}$ & Fixed & 45.38 & 0.16 & 1.071 & $0.891-1.287$ & 0.733 & 0.464 & 1 & 0.431 \\
\hline & TT vs. CC & Fixed & 8.4 & 0.336 & 0.996 & $0.637-1.559$ & -0.016 & 0.987 & 1 & 0.439 \\
\hline & TC vs. CC & Fixed & 27.78 & 0.25 & 1.197 & $0.930-1.540$ & 1.393 & 0.164 & 1 & 0.413 \\
\hline & $\mathrm{TT}+\mathrm{TC}$ vs. CC & Fixed & 44.89 & 0.163 & 1.162 & $0.912-1.480$ & 1.214 & 0.225 & 1 & 0.406 \\
\hline & TT vs. TC+CC & Fixed & 0 & 0.573 & 0.913 & $0.594-1.403$ & -0.415 & 0.678 & 1 & 0.47 \\
\hline \multirow[t]{5}{*}{ Asians } & T vs. C & Random & 77.58 & $\leq 0.001$ & 1.173 & $0.958-1.438$ & 1.542 & 0.123 & 0.427 & 0.119 \\
\hline & TT vs. CC & Random & 55.31 & 0.008 & 1.295 & $0.944-1.776$ & 1.602 & 0.109 & 0.854 & 0.53 \\
\hline & TC vs. CC & Random & 79.26 & $\leq 0.001$ & 0.967 & $0.705-1.325$ & -0.211 & 0.833 & 0.945 & 0.264 \\
\hline & $\mathrm{TT}+\mathrm{TC}$ vs. CC & Random & 82.2 & $\leq 0.001$ & 1.119 & $0.816-1.532$ & 0.697 & 0.486 & 0.427 & 0.116 \\
\hline & TT vs. TC+CC & Random & 86.09 & $\leq 0.001$ & 1.594 & $0.961-2.642$ & 1.806 & 0.071 & 0.582 & 0.924 \\
\hline \multicolumn{11}{|c|}{ IL-12B rs3212227 } \\
\hline \multirow[t]{5}{*}{ Overall } & C vs. A & Random & 81.69 & 0.001 & 1.076 & $0.783-1.479$ & 0.452 & 0.651 & 0.308 & 0.236 \\
\hline & CC vs. AA & Random & 54.75 & 0.085 & 1.33 & $0.988-1.790$ & 1.878 & 0.06 & 0.734 & 0.782 \\
\hline & CA vs. AA & Random & 82.52 & 0.001 & 1.119 & $0.696-1.800$ & 0.463 & 0.643 & 0.308 & 0.356 \\
\hline & $\mathrm{CC}+\mathrm{CA}$ vs. AA & Random & 83.68 & $\leq 0.001$ & 1.125 & $0.704-1.799$ & 0.492 & 0.623 & 0.308 & 0.317 \\
\hline & $\mathrm{CC}$ vs. $\mathrm{CA}+\mathrm{AA}$ & Random & 24.76 & 0.263 & 1.14 & $0.874-1.487$ & 0.969 & 0.333 & 0.734 & 0.946 \\
\hline \multirow[t]{5}{*}{ Asians } & C vs. A & Fixed & 0 & 0.87 & 1.166 & $0.988-1.377$ & 1.812 & 0.07 & NA & NA \\
\hline & CC vs. AA & Fixed & 0 & 0.932 & 1.323 & $0.941-1.860$ & 1.61 & 0.107 & NA & NA \\
\hline & CA vs. AA & Fixed & 0 & 0.454 & 1.349 & $1.032-1.762$ & 2.191 & 0.028 & NA & NA \\
\hline & $\mathrm{CC}+\mathrm{CA}$ vs. $\mathrm{AA}$ & Fixed & 0 & 0.594 & 1.34 & $1.041-1.725$ & 2.271 & 0.023 & NA & NA \\
\hline & $\mathrm{CC}$ vs. $\mathrm{CA}+\mathrm{AA}$ & Fixed & 0 & 0.507 & 1.086 & $0.807-1.461$ & 0.542 & 0.588 & NA & NA \\
\hline
\end{tabular}

NA, not applicable 
Table 4. Summary Risk Estimates for Association of IL-6 rs1800795, TNF- $\alpha$ rs 1800629 and TLR9 rs352140 Polymorphisms with Cervical Cancer Risk

\begin{tabular}{|c|c|c|c|c|c|c|c|c|c|c|}
\hline \multirow[t]{2}{*}{ Subgroup } & \multirow[t]{2}{*}{ Genetic Model } & \multirow{2}{*}{$\begin{array}{l}\text { Type of } \\
\text { Model }\end{array}$} & \multicolumn{2}{|c|}{ Heterogeneity } & \multicolumn{2}{|c|}{ Odds Ratio } & \multirow[b]{2}{*}{$Z_{\text {test }}$} & \multirow[b]{2}{*}{$\mathrm{P}_{\mathrm{OR}}$} & \multicolumn{2}{|c|}{ Publication Bias } \\
\hline & & & $\mathrm{I}^{2}(\%)$ & $\mathrm{P}_{\mathrm{H}}$ & OR & $95 \% \mathrm{CI}$ & & & $\mathrm{P}_{\text {Beggs }}$ & $P_{\text {Eggers }}$ \\
\hline \multicolumn{11}{|c|}{ IL-6 rs1800795 } \\
\hline \multirow[t]{5}{*}{ Overall } & C vs. G & Random & 60 & 0.02 & 1.294 & $1.071-1.564$ & 2.675 & 0.007 & 0.763 & 0.701 \\
\hline & CC vs. GG & Random & 54.07 & 0.042 & 1.633 & $1.059-2.520$ & 2.217 & 0.027 & 0.763 & 0.587 \\
\hline & CG vs. GG & Random & 52.47 & 0.049 & 1.232 & $0.971-1.562$ & 1.718 & 0.086 & 0.763 & 0.728 \\
\hline & $\mathrm{CC}+\mathrm{CG}$ vs. $\mathrm{GG}$ & Random & 53.01 & 0.047 & 1.312 & $1.048-1.643$ & 2.371 & 0.018 & 1 & 0.583 \\
\hline & $\mathrm{CC}$ vs. $\mathrm{CG}+\mathrm{GG}$ & Fixed & 48.97 & 0.068 & 1.592 & $1.268-1.999$ & 3.999 & $\leq 0.001$ & 0.548 & 0.646 \\
\hline \multirow[t]{5}{*}{ Asians } & C vs. G & Fixed & 65.97 & 0.053 & 1.395 & $1.230-1.582$ & 5.195 & $\leq 0.001$ & 1 & 0.793 \\
\hline & CC vs. GG & Fixed & 56.74 & 0.099 & 1.951 & $1.472-2.584$ & 4.656 & $\leq 0.001$ & 1 & 0.375 \\
\hline & CG vs. GG & Fixed & 66.54 & 0.05 & 1.289 & $1.077-1.543$ & 2.769 & 0.006 & 1 & 0.613 \\
\hline & $\mathrm{CC}+\mathrm{CG}$ vs. GG & Fixed & 61 & 0.077 & 1.429 & $1.207-1.692$ & 4.136 & $\leq 0.001$ & 1 & 0.766 \\
\hline & $\mathrm{CC}$ vs. $\mathrm{CG}+\mathrm{GG}$ & Fixed & 53.87 & 0.114 & 1.736 & $1.339-2.251$ & 4.166 & $\leq 0.001$ & 0.296 & 0.205 \\
\hline \multicolumn{11}{|c|}{ TNF- $\alpha$ rs 1800629} \\
\hline \multirow[t]{5}{*}{ Overall } & A vs. G & Random & 61.94 & $\leq 0.001$ & 1.277 & $1.104-1.477$ & 3.291 & 0.001 & 0.029 & 0.025 \\
\hline & AA vs. GG & Fixed & 27.43 & 0.125 & 1.333 & $1.062-1.674$ & 2.481 & 0.013 & 0.314 & 0.366 \\
\hline & AG vs. GG & Random & 70.89 & $\leq 0.001$ & 1.307 & $1.064-1.605$ & 2.552 & 0.011 & 0.183 & 0.141 \\
\hline & $\mathrm{AA}+\mathrm{AG}$ vs. $\mathrm{GG}$ & Random & 67.34 & $\leq 0.001$ & 1.324 & $1.104-1.587$ & 3.03 & 0.002 & 0.097 & 0.056 \\
\hline & AA vs. AG+GG & Fixed & 35.98 & 0.056 & 1.221 & $0.977-1.525$ & 1.758 & 0.079 & 0.537 & 0.336 \\
\hline \multirow[t]{5}{*}{ Asians } & A vs. G & Random & 78.48 & $\leq 0.001$ & 1.403 & $0.970-2.029$ & 1.798 & 0.072 & 0.035 & 0.062 \\
\hline & AA vs. GG & Fixed & 43.54 & 0.088 & 1.089 & $0.670-1.770$ & 0.343 & 0.731 & 1 & 0.54 \\
\hline & AG vs. GG & Random & 82.21 & $\leq 0.001$ & 1.469 & $0.895-2.411$ & 1.521 & 0.128 & 0.173 & 0.267 \\
\hline & $\mathrm{AA}+\mathrm{AG}$ vs. GG & Random & 81.63 & $\leq 0.001$ & 1.5 & $0.954-2.359$ & 1.756 & 0.079 & 0.173 & 0.121 \\
\hline & AA vs. AG+GG & Random & 50.71 & 0.048 & 1.04 & $0.487-2.217$ & 0.1 & 0.92 & 0.901 & 0.647 \\
\hline \multirow[t]{5}{*}{ Africans } & A vs. G & Fixed & 0 & 0.786 & 1.234 & $0.996-1.529$ & 1.925 & 0.054 & 1 & 0.739 \\
\hline & AA vs. GG & Fixed & 0 & 0.537 & 1.156 & $0.757-1.766$ & 0.672 & 0.502 & 1 & 0.289 \\
\hline & AG vs. GG & Fixed & 24.821 & 0.264 & 1.67 & $1.228-2.270$ & 3.268 & 0.001 & 1 & 0.564 \\
\hline & $\mathrm{AA}+\mathrm{AG}$ vs. $\mathrm{GG}$ & Fixed & 0 & 0.585 & 1.453 & $1.111-1.902$ & 2.725 & 0.006 & 1 & 0.766 \\
\hline & AA vs. AG+GG & Fixed & 0 & 0.702 & 0.955 & $0.640-1.425$ & -0.225 & 0.822 & 1 & 0.185 \\
\hline \multirow[t]{5}{*}{ Caucasians } & A vs. G & Random & 52.45 & 0.032 & 1.242 & $1.043-1.478$ & 2.438 & 0.015 & 0.754 & 0.203 \\
\hline & AA vs. GG & Fixed & 22.58 & 0.242 & 1.586 & $1.147-2.193$ & 2.791 & 0.005 & 0.175 & 0.072 \\
\hline & AG vs. GG & Random & 54.87 & 0.023 & 1.123 & $0.905-1.395$ & 1.056 & 0.291 & 0.754 & 0.906 \\
\hline & $\mathrm{AA}+\mathrm{AG}$ vs. $\mathrm{GG}$ & Random & 52.8 & 0.031 & 1.201 & $0.982-1.469$ & 1.787 & 0.074 & 0.916 & 0.501 \\
\hline & AA vs. AG+GG & Fixed & 22.15 & 0.246 & 1.569 & $1.137-2.165$ & 2.744 & 0.006 & 0.348 & 0.079 \\
\hline \multicolumn{11}{|c|}{ TLR9 rs352140 } \\
\hline \multirow[t]{5}{*}{ Overall } & A vs. G & Random & 84.19 & $\leq 0.001$ & 1.231 & $0.946-1.603$ & 1.545 & 0.122 & 0.763 & 0.892 \\
\hline & AA vs. GG & Random & 77.72 & $\leq 0.001$ & 1.341 & $0.834-2.154$ & 1.211 & 0.226 & 0.548 & 0.773 \\
\hline & AG vs. GG & Random & 57.65 & 0.028 & 1.236 & $0.962-1.588$ & 1.658 & 0.097 & 1 & 0.925 \\
\hline & $\mathrm{AA}+\mathrm{AG}$ vs. $\mathrm{GG}$ & Random & 72.99 & 0.001 & 1.299 & $0.967-1.745$ & 1.74 & 0.082 & 1 & 0.939 \\
\hline & AA vs. AG+GG & Random & 76.74 & $\leq 0.001$ & 1.226 & $0.810-1.856$ & 0.962 & 0.336 & 0.763 & 0.965 \\
\hline
\end{tabular}

ethnicity revealed an increased risk of cervical cancer in Asian women.

\section{TLR9 rs 352140}

The summary for the association of TLR9 rs352140 polymorphism with cervical cancer risk are shown in Table 4. Pooled results showed that the TLR9 rs352140 polymorphism was not associated with cervical cancer in overall population and by ethnicity.
Test of heterogeneity

In the current meta-analysis the $\chi^{2}$ test and $\mathrm{I}^{2}$ statistics were used for assessing the heterogeneity of the included studies. Results indicate that there was statistical heterogeneity for XRCC3 rs861539, MTHFR rs1801133, IL-6 rs1800795, IL-12B rs3212227, TNF- $\alpha$ rs1800629, and TLR9 rs352140 polymorphisms under most genetic models. Thus, the random effect model was used for evaluating the pooled OR and 95\% CI for those models. Subgroup analyses showed that ethnicity of participants 
might contribute to part of heterogeneity.

\section{Sensitivity Analysis}

The process of performing a meta-analysis involves a sequence of decisions and it is important to perform a sensitivity analysis in order to assess the impact of different decisions on pooled data. Thus, a sensitivity analysis was carried out by excluding a single study in turn on pooled ORs. The results showed that no individual study had an influence on the pooled OR all involved polymorphisms at XRCC3 rs861539, MTHFR rs1801133, IL-6 rs1800795, IL-12B rs3212227, TNF- $\alpha$ rs1800629, and TLR9 rs352140 polymorphisms, suggesting the stability of our findings. Moreover, excluding HWE deviated studies suggested that there were no independent studies that significantly influenced our pooled data.

\section{Publication Bias}

Begg's funnel plot and Egger's test were used for evaluating publication bias. As shown in Tables 3 and 4, the Egger's test results showed that there was no publication bias for the XRCC3 rs861539, MTHFR rs1801133, IL-6 rs1800795, IL-12B rs3212227, TNF- $\alpha$ rs1800629, and TLR9 rs352140 polymorphisms under all five genetic models. Moreover, Begg's funnel did not statistically revealed a significant publication bias in any of the models for all involved polymorphisms. Thus, the publication bias tests revealed that our pooled ORs were reliable.

\section{Discussion}

Genetic factors have been shown to influence the susceptibility of patients to various diseases and have attracted increasing attention (Motamedi et al., 2012; Mazaheri et al., 2014; Kabiri Rad et al., 2018). Several efforts have been made to identify the genetic susceptibility factors underlying development of cervical cancer. However, only a few polymorphisms have shown consistency among studies. In this meta-analysis, the association of XRCC3 rs861539, MTHFR rs1801133, IL-6 rs1800795, IL-12B rs3212227, TNF- $\alpha$ rs 1800629 and TLR9 rs352140 polymorphisms with susceptibility to cervical carcinoma was assessed by including all relevant studies.

Yuan et al., (2021) in a meta-analysis based on 15 studies with 5,740 cases and 9,931 controls revealed that there was no significant association between the XRCC3 Thr241Met and the risk of gynecological malignancies. However, their subgroup analysis by ethnicity showed that XRCC3 Thr241Met was associated with an increased risk of gynecological malignancies in Asians. Moreover, their stratified analysis by cancer type indicated that XRCC3 Thr241Met associated with cervical cancer in Asians (CT vs. $\mathrm{CC}$ : $\mathrm{OR}=1.50,95 \% \mathrm{CI}=1.04-2.14$; $\mathrm{TT}$ vs. $\mathrm{CC}: \mathrm{OR}=3.14$, 95\% $\mathrm{CI}=1.38-7.14 ; \mathrm{CT}+\mathrm{TT}$ vs. $\mathrm{CC}: \mathrm{OR}=1.64,95 \%$ $\mathrm{CI}=1.17-2.31)$. Abbas et al., (2010) in a case-control study with 260 cervical cancer cases and 265 controls evaluated the association of XRCC1+399A/G, XRCC2+31467G/A and XRCC3+18067C/T polymorphisms with cervical cancer in Indian women. Their results showed that XRCC2+31479G/A and XRCC3+18067C/T polymorphisms were not associated with cervical cancer. However, they showed that he XRCC $1+399 \mathrm{~A} / \mathrm{G}$ is linked with cervical cancer in the Indian population. Al-Harbi et al., (2017) in a study among 232 cervical cancer cases and 313 control subjects evaluated the association of CDKN1A C31A, ATM G1853A, HDM2 T309G, TGFB1 T10C, XRCC1 G399A, and XRCC3 C241T with cervical cancer among Saudi Arabian women. They showed that the TGFB1 T10C and XRCC1 G399A polymorphisms were associated with cervical cancer risk.

Our pooled results showed that the MTHFR rs1801133 polymorphism was not associated with cervical cancer in overall population and by ethnicity. Silva et al., (2019)reported that there were no differences in the genotypic and allelic distribution of MTHFR C677T polymorphism between remission (with the presence of pre-neoplastic lesions) and Persistence (with the presence of pre-neoplastic lesions). The same authors, in another study showed that MTHFR C677T polymorphism was not associated with cervical cancer and HPV infection (Silva et al., 2019). However, Sohrabi et al., in a case-control study evaluated the association of MTHFR A1298C and C677T variants among in 50 cervical intraepithelial neoplasia cases, 98 HPV-positive subjects and 47 non-cancerous/non-HPV patients as healthy controls. Their results showed that MTHFR $1298 \mathrm{CC}$ is more likely to be a potential risk factor for HPV-cervical cancer progression (Sohrabi et al., 2020). Zhou et al., (2020) indicated that the MTHFR rs4846048 enhanced the risk of cervical cancer through association with miR-522. Gong et al., (2018) reported that MTHFR C677T polymorphism was not associated with the risk of cervical cancer or cervical intra-epithelial neoplasia, while, the MTHFR A1298C polymorphism could increase the risk of both cervical cancer and cervical intra-epithelial neoplasia.

In the current study pooled data indicated that the TNF- $\alpha$ rs1800629 polymorphism was associated with cervical cancer risk. Wang et al., (2012) in meta-analysis based 27 studies showed that both TNF- $\alpha-238$ and -308 G/A polymorphisms could be used to identity individual with elevated susceptibility to cervical cancer in by ethnicity (Bi, 2014). Behboodi et al., (2021) in a study among 153 Iranian cervical cancer cases and 292 free cancer subjects demonstrated that TNF- $\alpha$ rs 1800629 was associated with increased level and risk of developing cervical cancer. However, Duvlis et al., (2020) reported that TNF-a-238G/A and TNF-a-308 G/T polymorphisms were not associated with the risk of HPV associated cervical intraepithelial lesions or cervical cancer cases in Macedonian women compared to controls. Moreover, Traore et al., (2020) revealed that TNF-308 G/A or IL18-607C/A polymorphisms were not associated with HPV infection among Burkina Faso women.

Pooled data from all eligible studies showed that the IL-6 rs1800795 polymorphism was significantly associated with cervical cancer risk in overall population and among Asian women. Similarly, Duan et al., (2018) in a meta-analysis based on 7 studies showed that the IL-6 rs1800795 polymorphism is associated with risk of cervical cancer in overall. Pu et al., (2016) in a study 
with 360 cervical cancer cases and 728 healthy subjects showed that this polymorphism is risk factor for cervical cancer development in Chinese women. de Souza et al., (2006) in a case-control study with 56 cases and 253 controls evaluated the association of IL-6 rs1800795 polymorphism with cervical cancer in a Brazilian population. In 2017, Zidi et al., (2017) in a case-control study evaluated the effects of six different genetic variants atIL-6 with risk of cervical cancer. The study revealed that the IL-6 rs1800795 polymorphism has a has a protective role in cervical cancer development in Tunisian women.de Lima Júnior et al., (2016) revealed that the IL-6 rs 1800795 polymorphism was not associated with HPV infection and healthy controls in in Brazilian women. The current meta-analysis data showed that the IL-12B rs3212227 genetic variant was not associated with susceptibility to cervical cancer, while; stratified analysis showed that this polymorphism was associated with cervical cancer risk among Asian women. Zheng et al., (2017) in a metaanalysis based on 33 articles with 10,587 cancer cases and 12,040 healthy subjects assessed the genetic association of IL-12B rs3212227 with cancer risk. Their pooled data indicated that IL-12B rs3212227 polymorphism was associated with cancer risk in overall. However, subgroup analysis by cancer type showed that IL-12B rs3212227 was not associated with cervical cancer. In 2016, Chang et al., (2015) in a meta-analysis based on 5 studies with 2552 cervical cases and 2232 healthy subjects showed that this polymorphism did not associate with risk of cervical cancer. In 2012, de Carvalho et al., found that the IL-12B rs3212227 variant has a protective role in development of cervical cancer in Brazilian women (Do Carmo Vasconcelos De Carvalho et al., 2012). Similarly, Han et al., (2008) found that IL-12B rs3212227 did not associate with cervical cancer risk in Korean population. However, Roszak et al., (2012) showed that this polymorphism was associated with increased risk of cervical cancer among polish women.

The current meta-analysis data showed that TLR9 rs352140 was not associated with susceptibility to cervical cancer. Nath et al., (2020) in a study showed that TLR4/9 polymorphisms are associated with increased HPV16/18 infection susceptibility and cervical squamous cell carcinoma risk among the women of Jharkhand state. In another study in India showed the TLR4 and TLR9 polymorphisms and haplotypes with hrHPV infection and cervical cancer risk (Pandey et al., 2019). MartínezCampos et al., (2017) in a case-control study revealed that TLR9 rs187084 is a risk factor for HPV infection, squamous intraepithelial cervical lesion, and uterine cervical neoplasm in Mexican female population. Jin et al., (2017) also evaluated the association of some variants at Toll-like receptors gene with cervical cancer among 420 Chinese cervical cancer patients and 842 controls. Their results showed that mutant alleles of TLR2 rs3775290, TLR4 rs7873784, and TLR9 rs352140 were associated with increased cervical cancer risk. Similarly, Yang et al., (2020) in meta-analysis based on eleven studies indicated that the TLR9 rs187084 and rs352140 polymorphisms may contribute to development of cervical cancer, but not TLR2-196 to -174 del/ins polymorphism.
In summary, our pooled data indicated that the XRCC3 RS861539, TNF- $\alpha$ rs1800629, and IL-6 rs1800795 genetic variants were associated with susceptibility to cervical cancer globally. However, the MTHFR rs1801133, IL-12B rs3212227 and TLR9 rs352140 variants were not associated. Larger and more rigorous studies among different ethnicities are needed to further evaluate these associations with cervical cancer.

\section{Author Contribution Statement}

Seyedeh Fatemeh Parsaeian, Fatemeh Asadian, Mojgan Karimi-Zarchi: conceptualization, investigation. Seyed Alireza Dastgheib, Sepideh Setayesh: Software, original draft preparation. Atiyeh Javaheri, Razieh Sadat Tabatabaie: Investigation. Fatemeh Asadian: Investigation, writing. Fatemeh Asadian, Hossein Neamatzadeh: Methodology, software. Hossein Golestanpour, Hossein Neamatzadeh: Formal analysis, investigation. Hossein Golestanpour: Project administration. Atiyeh Javaheri, Razieh Sadat Tabatabaie: Writing, reviewing, editing.

\section{Acknowledgements}

None.

\section{Ethics approval}

This article does not contain any studies with human participants or animals performed by any of the authors.

\section{Consent to participate}

Not applicable for this manuscript.

\section{Availability of data and material}

The datasets generated during and/or analyzed during this study are available from the corresponding author on reasonable request.

\section{Conflicts of interest}

The authors declare that they have no conflict of interest.

\section{References}

Abbas M, Srivastava K, Imran M, Banerjee M (2019). Genetic polymorphisms in DNA repair genes and their association with cervical cancer. Br J Biomed Sci, 76, 117-21.

Ahmadi SAY, Sayad S, Shahsavar F, et al (2021). Expression of Angiogenesis-related Genes in a Group of Iranian Cases of Breast Cancer. Curr Pharmacogenomics Person Med, 17, 197-205.

Al-Harbi NM, Bin Judia SS, Mishra KN, Shoukri MM, Alsbeih GA(2017). Genetic predisposition to cervical cancer and the association with XRCC1 and TGFB1 polymorphisms. Int $J$ Gynecol Cancer, 27, 1949-1956.

Antikchi MH, Neamatzadeh H, Ghelmani Y, et al (2021). The Risk and Prevalence of COVID-19 Infection in Colorectal Cancer Patients: a Systematic Review and Meta-analysis. $J$ Gastrointest Cancer, 52, 73-9.

Arbyn M, Weiderpass E, Bruni L, et al (2020). Estimates of incidence and mortality of cervical cancer in 2018: a worldwide analysis. Lancet Glob Health, 8, e191-e203.

Azadi-Yazdi M, Karimi-Zarchi M, Salehi-Abargouei A, 
Fallahzadeh H, Nadjarzadeh A (2017). Effects of Dietary Approach to Stop Hypertension diet on androgens, antioxidant status and body composition in overweight and obese women with polycystic ovary syndrome: a randomised controlled trial. J Hum Nutr Diet, 30, 275-83.

Badano I, Stietz SM, Schurr TG, et al (2012). Analysis of TNFa promoter SNPs and the risk of cervical cancer in urban populations of Posadas (Misiones, Argentina). J Clin Virol, 53, 54-59.

Baghestani AR, Shahmirzalou P, Sayad S, Akbari ME, Zayeri F (2018). Comparison cure rate models by DIC criteria in Breast Cancer data. Asian Pac J Cancer Prev, 19, 1601-6.

Barbisan G, Pérez LO, Contreras A, Golijow CD (2012). TNF- $\alpha$ and IL-10 promoter polymorphisms, HPV infection, and cervical cancer risk. Tumour Biol, 33, 1549-56.

Behboodi N, Farazestanian M, Rastgar-Moghadam A, et al (2021). Association of a variant in the tumor necrosis factor alpha gene with risk of cervical cancer. Mol Biol Rep, 48, 1433-7.

Bi X (2014). Study on TLRs polymorphisms and cervical cancer susceptibility. Progr Obstetrics Gynecol, 23, 520-3.

Binesh F, Zarchi MK, Vahedian H, Rajabzadeh Y (2012). Primary malignant lymphoma of the uterine cervix. BMJ Case Rep, 2012, bcr2012006675.

Calhoun ES, McGovern RM, Janney CA, et al (2002). Host genetic polymorphism analysis in cervical cancer. Clin Chem, 48, 1218-24.

Do Carmo Vasconcelos De Carvalho V, De MacÊdo JL, Albertina Dantas De Lima C, et al (2012). IFN-gamma and IL-12B polymorphisms in women with cervical intraepithellial neoplasia caused by human papillomavirus. Mol Biol Rep, 39, 7627-34.

Chang S-W, Xu G-Q, Fan Y-L (2015). Association of interleukin-12 gene polymorphisms with cancer susceptibility: a meta-analysis. Int $J$ Clin Exp Med, 8, 5317-22.

Chen D, Enroth S, Liu H, et al (2016). Pooled analysis of genome-wide association studies of cervical intraepithelial neoplasia 3 (CIN3) identifies a new susceptibility locus. Oncotarget, 7, 42216-24.

Chen X, Han S, Wang S, et al (2009). Interactions of IL-12A and IL-12B polymorphisms on the risk of cervical cancer in Chinese women. Clin Cancer Res, 15, 400-5.

Chen X, Jiang J, Shen H, Hu Z (2011). Genetic susceptibility of cervical cancer. J Biomed Res, 25, 155-64.

Dastgheib SA, Najafi F, Shajari A, et al (2020). Association of plasminogen activator inhibitor-1 4G5G Polymorphism with risk of diabetic nephropathy and retinopathy: a systematic review and meta-analysis. J Diabetes Metab Disord, 19, 2005-16.

Delgado-Enciso I, Martínez-Garza SG, Rojas-Martínez A, et al (2006). The effect of MTHFR polymorphisms, pregnancy and first intercourse on cervical cancer in a population from the Northeastern Mexico. Rev Invest Clin, 58, 462-9.

Deshpande A, Nolan JP, White PS, et al (2005). TNF- $\alpha$ promoter polymorphisms and susceptibility to human papillomavirus 16-associated cervical cancer. J Infect Dis, 191, 969-76.

Djansugurova LB, Perfilyeva AV, Zhunusova GS, Djantaeva KB, Iksan OA, Khussainova EM (2013). The determination of genetic markers of age-related cancer pathologies in populations from Kazakhstan. Front Genet, 4, 70.

Duan HX, Chen YY, Shi JZ, Ren NN, Li XJ (2018). Association of IL-6 -174G $>C$ (rs1800795) polymorphism with cervical cancer susceptibility. Biosci Rep, 38, BSR20181071.

Duarte I, Santos A, Sousa H, et al (2005). G-308A TNF- $\alpha$ polymorphism is associated with an increased risk of invasive cervical cancer. Biochem Biophys Res Commun,
334, 588-92.

Duvlis S, Dabeski D, Cvetkovski A, Mladenovska K, PlaseskaKaranfilska D (2020). Association of TNF-a (rs361525 and rs1800629) with susceptibility to cervical intraepithelial lesion and cervical carcinoma in women from Republic of North Macedonia. Int J Immunogenet, 47, 522-8.

Gangwar R, Mittal B, Mittal RD (2009). Association of interleukin-6 $-174 \mathrm{G}>\mathrm{C}$ promoter polymorphism with risk of cervical cancer. Int J Biol Markers, 24, 11-6.

Ghaemmaghami F, Zarchi MK, Gilani MM, et al (2008). Uterine sarcoma: Clinicopathological characteristics, treatment and outcome in Iran. Asian Pac J Cancer Prev, 9, 421-426.

Ghaemmaghami F, Zarchi MK, Mousavi A (2008). Surgical management of primary vulvar lymphangioma circumscriptum and postradiation: Case Series and Review of Literature. J Minim Invasive Gynecol, 15, 205-8.

Ghelmani Y, Asadian F, Antikchi MH, et al (2021). Association between the hOGG1 $1245 \mathrm{C}>\mathrm{G}$ (rs1052133) polymorphism and susceptibility to colorectal cancer: a Meta-analysis Based on 7010 Cases and 10,674 Controls. J Gastrointest Cancer, 52, 389-98.

Gong J-M, Shen Y, Shan W-W, He Y-X (2018). The association between MTHFR polymorphism and cervical cancer. $S_{c i}$ Rep, $\mathbf{8}, 7244$.

Gostout BS, Poland GA, Calhoun ES, et al (2003). TAP1, TAP2, and HLA-DR2 alleles are predictors of cervical cancer risk. Gynecol Oncol, 88, 326-32.

Govan VA, Constant D, Hoffman M, Williamson A-L (2006). The allelic distribution of -308 Tumor Necrosis Factor-alpha gene polymorphism in South African women with cervical cancer and control women. BMC Cancer, 6, 24.

Grimm C, Watrowski R, Baumühlner K, et al (2011). Genetic variations of interleukin-1 and -6 genes and risk of cervical intraepithelial neoplasia. Gynecol Oncol, 121, 537-41.

Hamadani S, Kamali M, Sedigheh Hantoushzadeh, Razieh Sadat Tabatabaee HN, et al (2017). Association of the s1799724 and rs 1800629 Polymorphisms of the TNF- $\alpha$ Gene with Susceptibility to Cervical Cancer: a Systematic Review and Meta-Analysis based on 24 Case-Control Studies. Asian Pac J Cancer Care, 2, 29-36.

Han SS, Cho EY, Lee TS, et al (2008). Interleukin-12 p40 gene (IL12B) polymorphisms and the risk of cervical caner in Korean women. Eur J Obstet Gynecol Reprod Biol, 140, 71-75.

He X, Ye F, Zhang J, et al (2008). Susceptibility of XRCC3, $\mathrm{XPD}$, and XPG genetic variants to cervical carcinoma. Pathobiology, 75, 356-63.

Ivansson EL, Juko-Pecirep I, Gyllensten UB (2010). Interaction of immunological genes on chromosome $2 \mathrm{q} 33$ and IFNG in susceptibility to cervical cancer. Gynecol Oncol, 116, 544-8.

Jafari M, Jarahzadeh MH, Dastgheib SA, et al (2020). Association of PAI-1 rs1799889 polymorphism with susceptibility to ischemic stroke: a Huge Meta-Analysis based on 44 Studies. Acta Medica (Hradec Kralove), 63, 31-42.

Jang WH, Yang YI, Yea SS, et al (2001). The -238 tumor necrosis factor-alpha promoter polymorphism is associated with decreased susceptibility to cancers. Cancer Lett, 166, 41-6.

Jarahzadeh MH, Asadian F, Farbod M, et al (2021). Cancer and Coronavirus Disease (COVID-19): Comorbidity, Mechanical Ventilation, and Death Risk. J Gastrointestinal Cancer, 52, 80-4.

Jin Y, Qiu S, Shao N, Zheng J (2017). Association of toll-like receptor gene polymorphisms and its interaction with HPV infection in determining the susceptibility of cervical cancer in Chinese Han population. Mamm Genome, 28, 213-9.

Kabiri Rad H, Mazaheri M, Dehghani Firozabadi A (2018). Relative expression of PBMC MicroRNA-133a analysis 
in patients receiving Warfarin after mechanical heart valve replacement. Avicenna J Med Biotechnol, 10, 29-33.

Kang S, Kim JW, Kang GH, et al (2005). Polymorphism in folate- and methionine-metabolizing enzyme and aberrant $\mathrm{CpG}$ island hypermethylation in uterine cervical cancer. Gynecol Oncol, 96, 173-80.

Karimi-Zarchi M, Dehghani-Firoozabadi R, Tabatabaie A, et al (2013). A comparison of the effect of levonorgestrel IUD with oral medroxyprogesterone acetate on abnormal uterine bleeding with simple endometrial hyperplasia and fertility preservation. Clin Exp Obstet Gynecol, 40, 421-4.

von Keyserling H, Bergmann T, Schuetz M, et al (2011). Analysis of 4 single-nucleotide polymorphisms in relation to cervical dysplasia and cancer development using a high-throughput ligation-detection reaction procedure. Int J Gynecol Cancer, 21, 1664-71.

Kohaar I, Kumar J, Thakur N, et al (2010). Homocysteine levels are associated with cervical cancer independent of methylene tetrahydrofolate reductase gene (MTHFR) polymorphisms in Indian population. Biomarkers, 15, 61-8.

Kohaar I, Thakur N, Salhan S, et al (2007). TNFalpha-308G/A polymorphism as a risk factor for HPV associated cervical cancer in Indian population. Cell Oncol, 29, 249-56.

Lai ZZ, Ni-Zhang, Pan XL, Song L (2013). Toll-like receptor 9 (TLR9) gene polymorphisms associated with increased susceptibility of human papillomavirus-16 infection in patients with cervical cancer. $J$ Int Med Res, 41, 1027-36.

Lambropoulos AF, Agorastos T, Foka ZJ, et al (2003). Methylenetetrahydrofolate reductase polymorphism C677T is not associated to the risk of cervical dysplasia. Cancer Lett, 191, 187-91.

Leo PJ, Madeleine MM, Wang S, et al (2017). Defining the genetic susceptibility to cervical neoplasia-A genome-wide association study. PLoS Genet, 13, e1006866.

de Lima Júnior SF, Tavares MMF, de Macedo JL, et al (2016). Influence of IL- 6 , IL- 8 , and TGF- $\beta 1$ gene polymorphisms on the risk of human papillomavirus-infection in women from Pernambuco, Brazil. Mem Inst Oswaldo Cruz, 111, 663-9.

Ma H, Jin G, Hu Z, et al (2006). Variant genotypes of CDKN1A and CDKN1B are associated with an increased risk of breast cancer in Chinese women. Int $J$ Cancer, 119, 2173-8.

Martínez-Campos C, Bahena-Román M, Torres-Poveda K, Burguete-García AI, Madrid-Marina V (2017). TLR9 gene polymorphism -1486T/C (Rs187084) is associated with uterine cervical neoplasm in Mexican female population. $J$ Cancer Res Clin Oncol, 143, 2437-45.

Mazaheri M, Shahdadi V, Boron AN (2014). Molecullar and biochemical effect of alcohlic extract of alpinia galanga on rat spermatogenesis process. Iran J Reprod Med, 12, 765-70.

Mirjalili H, Ezzabadi AR, Yazdiyousefi Y, Nadoshan MAJ (2019). The value of ultrasonography in determining pupillary light reflex in patients with traumatic ocular injuries; a Letter to Editor. Arch Acad Emerg Med, 7, 1-2.

Mostowska A, Myka M, Lianeri M, Roszak A, Jagodziński PP (2011). Folate and choline metabolism gene variants and development of uterine cervical carcinoma. Clin Bioch, 44, 596-600.

Motamedi S, Majidzadeh K, Mazaheri M, et al (2012). Tamoxifen resistance and CYP2D6 copy numbers in breast cancer patients. Asian Pac J Cancer Prev, 13, 6101-4.

Nandan NK, Wajid S, Biswas S, et al (2008). Allelic variations in 5,10-methylenetetrahydrofolate reductase gene and susceptibility to cervical cancer in Indian women. Drug Metab Lett, 2, 18-22.

Nath N, Mishra P, Panda AK, Mishra R (2020). Polymorphisms and haplotypes of TLR4, TLR9 and CYP1A1 genes possibly interfere with high-risk human papillomavirus infection and cervical cancer susceptibility in Jharkhand, India. Int Immunopharmacol, $\mathbf{8 8}$.

Nogueira De Souza NC, Brenna SMF, et al (2006). Interleukin-6 polymorphisms and the risk of cervical cancer. Int J Gynecol Cancer, 16, 1278-82.

Pal A, Kundu R (2020). Human papillomavirus E6 and E7: The Cervical Cancer Hallmarks and Targets for Therapy. Front Microbiol, 10, 3116.

Pandey NO, Chauhan AV, Raithatha NS, et al (2019). Association of TLR4 and TLR9 polymorphisms and haplotypes with cervical cancer susceptibility. Sci Rep, 9, 9729.

Pandey S, Mittal B, Srivastava M, et al (2011). Evaluation of Toll-like receptors 3 (c.1377C/T) and 9 (G2848A) gene polymorphisms in cervical cancer susceptibility. Mol Biol Rep, 38, 4715-21.

Pérez LO, Crivaro A, Barbisan G, Poleri L, Golijow CD (2013). XRCC2 R188H (rs3218536), XRCC3 T241M (rs861539) and R243H (rs77381814) Single Nucleotide polymorphisms in cervical cancer risk. Pathol Oncol Res, 19, 553-8.

Prasad VVTS, Wilkhoo H (2011). Association of the functional polymorphism C677T in the methylenetetrahydrofolate reductase gene with colorectal, thyroid, breast, ovarian, and cervical cancers. Onkologie, 34, 422-6.

$\mathrm{Pu}$ X, Gu Z, Wang X (2016). Polymorphisms of the interleukin 6 gene and additional gene-gene interaction contribute to cervical cancer susceptibility in Eastern Chinese women. Arch Gynecol Obstet, 294, 1305-10.

Rader JS, Tsaih SW, Fullin D, et al (2019). Genetic variations in human papillomavirus and cervical cancer outcomes. Int J Cancer, 144, 2206-14.

Roszak A, Lianeri M, Sowińska A, Jagodziński PP (2012). Involvement of toll-like receptor 9 polymorphism in cervical cancer development. Mol Biol Rep, 39, 8425-30.

Roszak A, Misztal M, Sowińska A, Jagodziński PP (2015). TNF- $\alpha-308 \mathrm{G} / \mathrm{A}$ as a risk marker of cervical cancer progression in the Polish population. Mol Diagn Ther, 19, 53-7.

Roszak A, Mostowska A, Sowińska A, Lianeri M, Jagodziński PP (2012). Contribution of IL12A and IL12B polymorphisms to the risk of cervical cancer. Pathol Oncol Res, 18, 997-1002.

Sayad S, Ahmadi SAY, Moradi M, et al (2020). A meta-analysis on diagnostic accuracy of serum HLA-G level in breast cancer. Expert Rev Precis Med Drug Dev, 5, 109-14.

Sayad S, Ahmadi SAY, Nekouian R, Panahi M, Anbari K (2020). Epidemiological and pathological characteristics of post-surgical cases of invasive breast cancer among ethnicities of Iran in 2018: A single center cross-sectional study. Arch Oncol, 26, 6-9.

Settheetham-Ishida W, Yuenyao P, Natphopsuk S, Settheetham D, Ishida T (2011). Genetic risk of DNA repair gene polymorphisms (XRCC1 and XRCC3) for high risk human papillomavirus negative cervical cancer in Northeast Thailand. Asian Pac J Cancer Prev, 12, 963-6.

Shekari M, Sobti RC, Kordi Tamandani DM, Suri V (2008). Impact of methylenetetrahydrofolate reductase (MTHFR) codon (677) and methionine synthase (MS) codon (2756) on risk of cervical carcinogenesis in North Indian population. Arch Gynecol Obstet, 278, 517-24.

Shi TY, Zhu ML, He J, et al (2013). Polymorphisms of the Interleukin 6 gene contribute to cervical cancer susceptibility in Eastern Chinese women. Hum Genet, 132, 301-12.

Da Silva M, De Albuquerque B, Allyrio T, et al (2021). The role of HPV-induced epigenetic changes in cervical carcinogenesis (Review). Biomed Rep, 15, 60.

Silva NNT, Sabino ADP, Tafuri A, Lima AA (2019). Lack of association between methylenetetrahydrofolate reductase C677T polymorphism, HPV infection and cervical 
intraepithelial neoplasia in Brazilian women. BMC Med Genet, 20, 100.

Singh H, Jain M, Sachan R, Mittal B (2009). Association of TNFA (-308G\&gt;A) and IL-10 (-819C\&gt; T) promoter polymorphisms with risk of cervical cancer. Int J Gynecol Cancer, 19, 1190-1194.

Soheili F, Nikkho B, Khodabandehloo M (2016). Determination of the physical status (Episomal/Integral) of HPV by qPCR in esophageal squamous cell carcinoma. Int $J$ Enteric Pathog, 5, 1-4.

Sohrabi A, Bassam-Tolami F, Imani M (2020). The impact of MTHFR $1298 \mathrm{~A}>\mathrm{C}$ and $677 \mathrm{C}>\mathrm{T}$ gene polymorphisms as susceptibility risk factors in cervical intraepithelial neoplasia related to HPV and sexually transmitted infections. J Obstet Gynaecol India, 70, 503-9.

Sousa H, Oliveira S, Santos AM, et al (2014). Tumour necrosis factor alpha $308 \mathrm{G} / \mathrm{A}$ is a risk marker for the progression from high-grade lesions to invasive cervical cancer. Tumo Biol, 35, 2561-4.

Stanczuk GA, Sibanda EN, Tswana SA, Bergstrom S (2019). Polymorphism at the -308-promoter position of the tumor necrosis factor-alpha (TNF-alpha) gene and cervical cancer. Int J Gynecol Cancer, 13, 148-53.

Sull JW, Jee SH, Yi S, et al (2004). The effect of methylenetetrahydrofolate reductase polymorphism C677T on cervical cancer in Korean women. Gynecol Oncol, 95, 557-63.

Tong S, Kim MK, Lee JK, et al (2011). Common polymorphisms in methylenetetrahydrofolate reductase gene are associated with risks of cervical intraepithelial neoplasia and cervical cancer in women with low serum folate and vitamin B12. Cancer Causes Control, 22, 63-72.

Traore IMA, Zohoncon TM, Djigma FW, et al (2020). Association of TNF- $\alpha-308 \mathrm{G} / \mathrm{A}$ and IL-18 Polymorphisms with risk of HPV infection among sexually active women in Burkina Faso. Biomol Concepts, 11, 97-101.

Wang N, Yin D, Zhang S, et al (2012). TNF-Alpha rs1800629 polymorphism is not associated with HPV infection or cervical cancer in the Chinese population. PLoS One, 7, e45246.

Wang Q, Zhang C, Walayat S, Chen HW, Wang Y (2011). Association between cytokine gene polymorphisms and cervical cancer in a Chinese population. Eur J Obstet Gynecol Reprod Biol, 158, 330-3.

Xiao H (2009). The association between XRCC3 gene polymorphism and cervical cancer risk. Jilin Med, 31, 2731-2.

Yang S, Liu L, Xu D, Li X (2020). The relationship of the TLR9 and TLR2 genetic polymorphisms with cervical cancer risk: a Meta-Analysis of Case-Control Studies. Pathol Oncol Res, 26, 307-15.

Yang F, Zhou Y, Jiang Y (2011). Study on the correlation between polymorphism of MTHFR gene and the pathogenesis of cervical cancer. Mat Child Health Care China, 26, 4087-9 (article in Chinese).

Yi Y, Fang Y, Wu K, Liu Y, Zhang W (2020). Comprehensive gene and pathway analysis of cervical cancer progression. Oncol Lett, 19, 3316-3332.

Yuan X, Wang Q, He G, Yu H (2021). Association between XRCC3 Thr241Met polymorphism and risk of gynecological malignancies: A meta-analysis. Cancer Genet, 254-255, $11-7$.

Zarchi MK, Akhavan A, Gholami H, et al (2010). Evaluation of cervical cancer risk-factors in women referred to Yazd-Iran hospitals from 2002 to 2009. Asian Pac J Cancer Prev, 11, 537-8.

Zheng Y, Wang M, Tian T, et al (2017). Role of interleukin-12 gene polymorphisms in the onset risk of cancer: A metaanalysis. Oncotarget, 8, 29795-807.

Zhou X, Shan L, Na J, Li Y, Wang J (2020). The SNP rs4846048 of MTHFR enhances the cervical cancer risk through association with miR-522: A preliminary report. Mol Genet Genomic Med, 8, e1055.

Zidi S, Sghaier I, Gazouani E, Mezlini A, Yacoubi-Loueslati B (2016). Evaluation of Toll-Like Receptors 2/3/4/9 Gene Polymorphisms in Cervical Cancer Evolution. Pathol Oncol Res, 22, 323-30.

Zidi S, Stayoussef M, Alsaleh BL, et al (2017). Relationships between common and novel interleukin- 6 gene polymorphisms and risk of cervical cancer: a Case-Control Study. Pathol Oncol Res, 23, 385-92.

Zidi S, Stayoussef M, Zouidi F, et al (2015). Tumor Necrosis Factor Alpha (-238/ -308) and TNFRII-VNTR (-322) Polymorphisms as Genetic Biomarkers of Susceptibility to Develop Cervical Cancer Among Tunisians. Pathol Oncol Res, 21, 339-45.

Zoodsma M, Nolte IM, Schipper M, et al (2005). Methylenetetrahydrofolate reductase (MTHFR) and susceptibility for (pre)neoplastic cervical disease. Hum Genet, 116, 247-54.

Zu F, Ai X, La L, Wu S, Gu Z (2010). A preliminary study of TNFa gene 308 single nucleotide polymorphism with cervical cancer risk and HPV subtype infection in southern Xinjiang Uygur patients [Article in Chinese]. Chung Hua Fu Chan Ko Tsa Chih, 45, 709-11.

Zuo F, Liang W, Ouyang Y, et al (2011). Association of TNF- $\alpha$ gene promoter polymorphisms with susceptibility of cervical cancer in Southwest China. Lab Med, 42, 287-90.

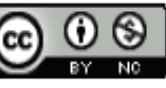

This work is licensed under a Creative Commons AttributionNon Commercial 4.0 International License. 